\title{
Frontotemporal Regulation of Subjective Value to Suppress Impulsivity in Intertemporal Choices
}

\author{
Stefan Dürschmid, ${ }^{1,2 *}$ Andre Maric, ${ }^{2 *}$ Marcel S. Kehl, ${ }^{3}$ Robert T. Knight, ${ }^{4}$ Hermann Hinrichs, ${ }^{1,2,5,6,7}$ and \\ Hans-Jochen Heinze H,2,5,6,7 $^{1}$ \\ ${ }^{1}$ Department of Behavioral Neurology, Leibniz Institute for Neurobiology, Magdeburg, 39120, Germany, ${ }^{2}$ Department of Neurology, Otto-von- \\ Guericke University, Magdeburg, 39120, Germany, ${ }^{3}$ Department of Epileptology, University Hospital Bonn, Bonn, 53127, Germany, ${ }^{4}$ Helen Wills \\ Neuroscience Institute and Department of Psychology, University of California, Berkeley, California 94720, ${ }^{5}$ German Center for Neurodegenerative \\ Diseases, Magdeburg, 39120, Germany, ${ }^{6}$ Forschungscampus STIMULATE, Otto-von-Guericke University, Magdeburg, 39106, Germany, and ${ }^{7}$ Center \\ of Behavioral Brain Sciences, Otto-von-Guericke University, Magdeburg, 39106, Germany
}

Impulsive decisions arise from preferring smaller but sooner rewards compared with larger but later rewards. How neural activity and attention to choice alternatives contribute to reward decisions during temporal discounting is not clear. Here we probed (1) attention to and (2) neural representation of delay and reward information in humans (both sexes) engaged in choices. We studied behavioral and frequency-specific dynamics supporting impulsive decisions on a fine-grained temporal scale using eye tracking and MEG recordings. In one condition, participants had to decide for themselves but pretended to decide for their best friend in a second prosocial condition, which required perspective taking. Hence, conditions varied in the value for themselves versus that pretending to choose for another person. Stronger impulsivity was reliably found across three independent groups for prosocial decisions. Eye tracking revealed a systematic shift of attention from the delay to the reward information and differences in eye tracking between conditions predicted differences in discounting. High-frequency activity $(175-250 \mathrm{~Hz})$ distributed over right frontotemporal sensors correlated with delay and reward information in consecutive temporal intervals for high value decisions for oneself but not the friend. Collectively, the results imply that the high-frequency activity recorded over frontotemporal MEG sensors plays a critical role in choice option integration.

Key words: delay discounting; eye tracking; frontal cortex; high-frequency activity; impulsivity; MEG

Significance Statement

Humans face decisions between sooner smaller rewards and larger later rewards daily. An objective benefit of losing weight over a longer time might be devalued in face of ice cream because they prefer currently available options because of insufficiently considering long-term alternatives. The degree of contribution of neural representation and attention to choice alternatives is not clear. We investigated correlates of such decisions in participants deciding for themselves or pretending to choose for a friend. Behaviorally participants discounted less in self-choices compared with the prosocial condition. Eye movement and MEG recordings revealed how participants represent choice options most evident for options with high subjective value. These results advance our understanding of neural mechanisms underlying decision-making in humans.

\section{Introduction}

Reward value decreases as a function of time: the longer we have to wait, the less a reward is typically valued. Hence,

\footnotetext{
Received May 11, 2020; revised Nov. 6, 2020; accepted Nov. 12, 2020.

Author contributions: S.D. and R.T.K. designed research; S.D. and A.M. performed research; S.D. and A.M. analyzed data; S.D., M.S.K., and R.T.K. wrote the first draft of the paper; S.D., M.S.K., R.T.K., H.H., and H.-J.H. edited the paper; S.D. and R.T.K. wrote the paper.

This work was supported by National Institute of Neurological Disorders and Stroke Grant R37NS21135, DFG/SFB 779 TP A02, Autonomie im Alter (EFRE).

*S.D and A.M. contributed equally to the study.

The authors declare no competing financial interests.

Correspondence should be addressed to Stefan Dürschmid at sduersch@lin-magdeburg.de.

https://doi.org/10.1523/JNEUROSCI.1196-20.2020

Copyright $\odot 2021$ the authors
}

delayed delivery of a larger reward converts an objective value into a perceived lesser value. This results in choosing a smaller but sooner (SS) rather than a larger but later reward (LL), a phenomenon known as delay discounting (DD). Impulsive decisions might be because of the tendency to prefer SS rewards compared with LL rewards. Previous fMRI studies focused on neuroanatomical correlates of subjective valuation, and observed interactions of multiple independent valuation systems in the ventromedial PFC (vmPFC) and the dorsolateral PFC (dlPFC) (McClure et al., 2004, 2007) where goal values correlate with vmPFC activity and the amount of self-control with dlPFC activity (Hare et al., 2009). Impulsivity might also result from insufficiently considered objective alternatives (Ainslie, 1975; Myerson et al., 
A

Experiment I: Reaction time
B

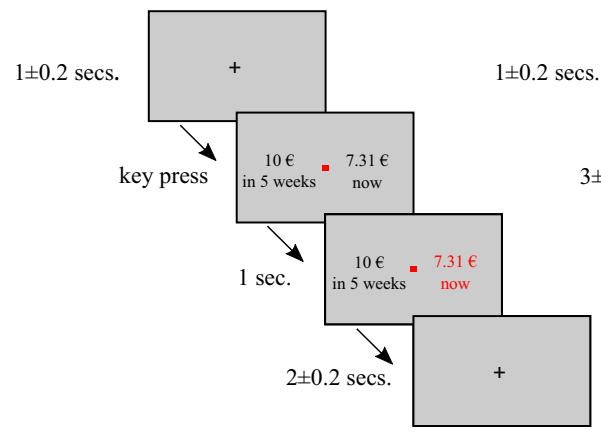

Experiment II: Eye tracker

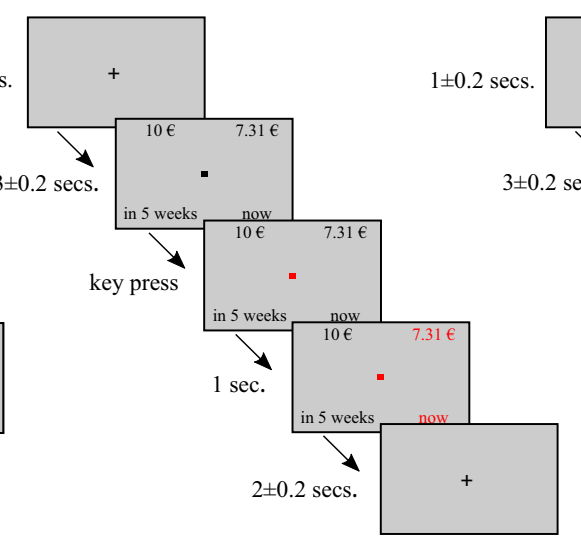

Experiment III: MEG

c 
A

CI Reaction time:

B
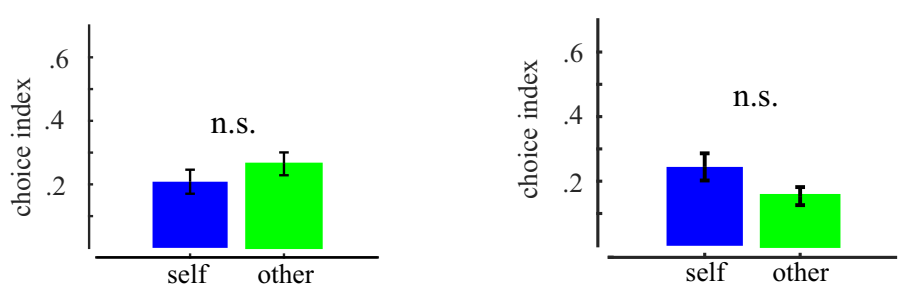

C

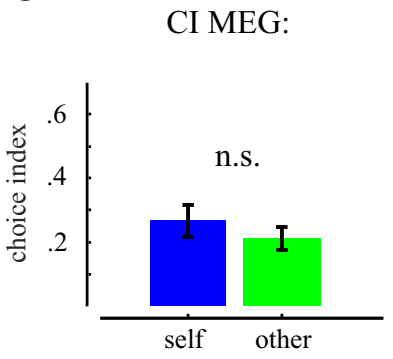

D

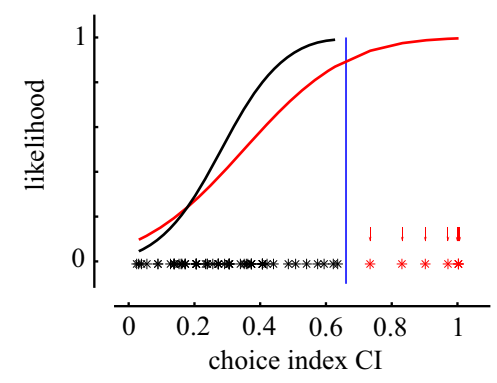

Figure 2. Cl distribution. A-C, Participants chose LL and SS options equally often and did not differ between conditions. Error bars indicate SEM. $\boldsymbol{D}$, Distribution of choice indices over all experiments with our cutoff at $\mathrm{Cl} \geq 0.66$

(Lockwood et al., 2017). All participants made their decisions in both conditions in separate blocks in counterbalanced order. Before blocks started, participants were instructed whether they have to make their decisions for themselves (SELF) or whether they had to pretend to decide for their best friend (OTHER). Participants were instructed before the experiment that, on completion of the experiment, one trial is randomly chosen from all presented choices (SELF and OTHER) and the respective monetary reward will be paid at the respective delay to avoid the belief that outcomes were only hypothetical. Participants were informed that this trial will be chosen randomly from both the SELF and OTHER condition to avoid biasing decisions to one of the two conditions. Prosocial behavior is commonly assessed as the willingness to benefit others. Altruism adds that this benefit has to be at their own costs. Most, if not all, theories of reciprocity, altruism, or prosocial behavior start from the assumption that perspective taking is the starting point of each social act. Our critical experimental condition is the instruction to assume the perspective of their best friend and to pretend to perform an act that (hypothetically) benefits others.

\section{General analysis}

This paradigm only allows a limited number of trials before participants arrive at their indifference point. In accord with previous studies, we used 60 trials. In addition, especially in MEG recordings, trials have to be rejected often because of artifacts. In participants with a strong trend to choose only one option across trials, this reduction would affect the option with fewer trials disproportionately stronger. As a result, averaged MEG responses could be largely determined by a prepotent motor response but not signals related to decision, especially in the OTHER condition in which we assumed that objective choice alternatives are less considered. To compare results across groups, we applied the criterion to all participants in all experiments. To identify participants with a strong trend to choose only one option across trials, we calculated a choice index $(C I)$ as follows:

$$
C I=\left|\frac{N_{S S}-N_{L L}}{N_{S S}+N_{L L}}\right|
$$

With $N_{S S}$ denoting the number of SS choices and $N_{L L}$ denoting the number of LL choices. Participants with $C I \geq 0.66$, that is, one option was chosen almost 5 times as often as the other option, were excluded (see Fig. 2) in both conditions. We used the average across the last 10 updated $\ln (k)$ parameters separately as proxy for individual indifference point in SELF and OTHER decisions. A higher $k$ value indicates steeper discounting of delayed rewards and thus more impulsive choices. As the distribution of discount rates is highly rightskewed, we used $\log$-transformed $k(\ln k)$ in all statistical analyses.

Statistical analysis. To correct statistical significance for multiple comparisons, we compared each statistical parameter against a surrogate distribution, which was constructed by randomly yoking labels of the trials and repeating the ANOVA, $t$ tests, or Pearson's correlation coefficient. Consequently, reported $p$ values represent the statistical significance relatively to the constructed surrogate distribution. All permutations (see detailed information for each test below) were conducted with MATLAB, and each surrogate distribution was constructed by running 1000 label permutations yielding 1000 surrogate values against which the observed statistical parameters were compared. Significance criterion in all these tests was statistical parameters corresponding with $p<0.025$. These critical values represent the $97.5 \%$ confidence and are denoted as $F_{\text {crit }}$, $T_{\text {crit }}$, or $r_{\text {crit. }}$. This approach counters the possibility that MEG and/or eye tracking data might be differently distributed. In general, bootstrap methods have the advantage of accounting for the dependence structure of $p$ values. Bootstrapping offers a way to deal with situations in which the test statistic may not follow the distribution assumed by large sample theory.

Power of correlation analysis. We conducted individual correlation analyses (e.g., gaze stability in eye tracking vs discount parameter or MEG activity vs discount parameter) where the number of participants has to be high to avoid false positives. Too few participants might not correctly reflect a small correlation leading to the false assumption that there is no correlation despite a de facto but low (e.g., $\rho=0.3$ ) correlation at the population level. Hence, a high number of subjects is needed for low correlation strength, but fewer participants can correctly reveal higher correlations at the population level (e.g., $\rho=0.6$ ). Increasing the number of participants without changing mean and SD decreases the $p$ value and hence increases the likelihood of exaggerating small effects. The number of participants used in our study are a trade-off between both approaches: a well-powered individual differences analysis without inflating mean effects. We determined the power of the maximal correlation coefficient $r$ using G*Power 3.1 (Erdfelder et al., 2009). The power values are given as $\beta$ separately for each analysis.

\section{Reaction time experiment}

Choice options were presented on the computer screen spanning a visual field with a horizontal visual angle of $9.5^{\circ}$ and vertical visual angle of $1.4^{\circ}$. Participants were instructed to closely evaluate the choice options and press one of two buttons indicating their choice when they had the feeling that they made their decision. If they did not respond following $10 \mathrm{~s}$, the next trial started (see Fig. 1A). We compared mean decision time and $\ln (k)$ parameters across participants between conditions using separate paired $t$ tests (see Fig. $3 A, B$ ).

\section{Eye tracking experiment}

Participants were instructed to withhold decision until the fixation point turned red ( $3 \mathrm{~s} \pm 200 \mathrm{~ms}$ following presentation of choice options; sufficiently long to exceed mean decision time in the first experiment) to eliminate any impact of button presses (see Fig. 1B). Choice options were presented further away from each other spanning an area of $41 \times$ $15.5 \mathrm{~cm}$ (horizontal visual angle of $32^{\circ}$ and vertical visual angle of $12^{\circ}$ ) to clearly distinguish gaze direction to the delay and reward. We used for eye movement recording the Eyelink 1000 system operated on Windows 7 and a desktop-mounted Eyelink CL camera with a TV lens $(35 \mathrm{~mm}$ 1:1.6). All participants used a chin and forehead rest with $71.5 \mathrm{~cm}$ 
distance to the monitor and $59 \mathrm{~cm}$ to the camera. Visual stimuli were presented on a Samsung Syncmaster $2233(22$ inch) with a resolution of $1680 \times 1050$. In each participant, we tracked the pupil diameter and corneal reflex of the left eye with a sampling rate of $2000 \mathrm{~Hz}$. Before each trial block, we performed a calibration session with the built-in 9-point grid method.

Data preprocessing. The resulting eye tracking data (time series of vertical eye movements to the reward presented above and delay below fixation cross) were used to characterize temporal dynamics of evaluation of delay and monetary reward. First, we identified trials $(-1$ to $4 \mathrm{~s}$ around stimulus onset) with low fixation in the baseline period $(-1 \mathrm{~s}$ to stimulus onset). That is, we calculated for each trial the mean $y$ coordinate (vertical eye movements between reward and delay). Trials with a mean $y$ value $>2$ SDs (indicating low fixation) of all trials were excluded from analyses. Since delay and reward were always presented below and above fixation, respectively (LL and SS options were presented pseudorandomly to the left and right), we focused on analyzing $y$ coordinates across time. We baseline-corrected by subtracting from each data point ( $y$ coordinate) the mean $y$ coordinate within the $-1 \mathrm{~s}$ preceding the stimulus onset in each trial. To define gaze direction as a function of time across trials, we then calculated at each time point a histogram of all $y$ coordinates (see Fig. $4 B$ ) separately for each participant. This results in high probability values for fixation before stimulus onset and high probability values for delay and reward following stimulus onset. These can be identified as colored bands in front of an otherwise dark blue background (locations on the screen where participants did not look at consistently) in Figure $4 B$. From these probability maps, we extracted three time series defined by gaze to delay $D_{t}$, to fixation $F_{t}$, and monetary reward $R_{t}$, representing time varying probability to look at delay, fixation, and reward, respectively. Since participants did not look exactly at one location, we defined a spatial margin around each of the three spatial regions based on the real eye movements. To this end, individual probability maps were averaged across participants and conditions and across time, leading to three probability maxima, which correspond with spatial location of delay, fixation, and reward (see Fig. $4 B$, right, dashed lines). The margin around these maxima is defined by the inflection points (solid lines). Probability values around the three maxima corresponding with spatial location on the screen (see Fig. 4B) were averaged at each time point, leading to three time series for each participant and condition. Figure $4 B$ (bottom) shows the time series for delay and reward.

Data analysis. Discounting differences. First, we compared $\ln (k)$ parameters across participants to test whether participants discounted differently between conditions (see Fig. $4 A$ ) with a paired $t$ test.

Intervals of option evaluation. To test for difference of gaze direction, we conducted a $t$ test at each time point between $D_{t}$ and $R_{t}$ time series across participants, leading to $T_{D R}$ time series. This time series shows when participants inspected on average more the delay or the reward. The level of significance of $\mathrm{T}_{\mathrm{DR}}$ was corrected for multiple comparisons by comparing each $\mathrm{T}_{\mathrm{DR}}$ value against a surrogate distribution. This surrogate distribution was constructed by randomly reassigning the labels (delay vs reward) to the single participants in 1000 permutations. This leads to 1000 surrogate $T_{D R}$ value time series. Significance criterion was a $\mathrm{T}_{\mathrm{DR}}$ value with $p<0.025$ within the surrogate distribution of all $T$ values (see Fig. $4 B$ ).

Differences in option evaluation. We tested whether participants evaluated choice options differently in both conditions. To test for difference of gaze direction between conditions, we conducted a $t$ test at each time point for $\mathrm{F}_{\mathrm{t}}, \mathrm{D}_{\mathrm{t}}$, and $\mathrm{R}_{\mathrm{t}}$ across participants, leading to three $t$ value time series $\left(T_{F}, T_{D}\right.$, and $\left.T_{R}\right)$ capturing the differences between conditions. The level of significance of each of the three $t$ value time series was corrected for multiple comparisons by comparing each $t$ value time series against a surrogate distribution as above but swapping labels (SELF vs OTHER). Significance criterion was a $t$ value with $p<0.025$ within the surrogate distribution of all surrogate $t$ values (see Fig. 4C).

Correlation of gaze entropy and decision. We hypothesized that differences in decision-making result from differences in choice evaluation. $D_{t}$ and $R_{t}$ time series representing the probability to look at the delay and the reward were used to estimate the entropy, which gives the average of information of all events (here gaze at delay and reward) and reflects the predictability or stability of gaze direction. If gaze entropy is high, it is hard to predict whether participants look at the delay or the reward; but if gaze entropy is low, then gaze direction is stable and prediction is high. In both conditions, entropy was calculated at each time point $t$ as follows:

$$
H_{t}=\sum_{i}^{m}-p_{i, t} \cdot \log _{2} p_{i, t}
$$

With $i$ denoting the reward and delay, $p$ as the likelihood to direct gaze at one of the two events. The resulting entropy time series $\mathrm{H}_{\mathrm{OTHER}}$ was subtracted from $\mathrm{H}_{\text {SELF}}$, leading to an $\mathrm{H}_{\Delta}$ time series for each participant. Individual differences in discount parameters were correlated with $\mathrm{H}_{\Delta}$ values at each time point, leading to a new Pearson's correlation $r$ time series. The level of significance of $r$ was corrected for multiple comparisons by comparing each $r$ against a surrogate distribution. This surrogate distribution was constructed in the following way. For each iteration, we randomly assigned the individual entropy values $\mathrm{H}_{\Delta}$ across participants. We then correlated these randomly assigned values with the individual discount values of our participant in 1000 permutations. This leads to 1000 surrogate $r$ value time series. Significance criterion was a $r$ value with $p<0.025$ within the surrogate distribution of all $r$ values (see Fig. 4D).

\section{MEG recordings}

Data acquisition. In a third group, participants $(N=24 ; 7$ female; mean age: $26.17 ; \mathrm{SD}=5.16$ ) were seated in a magnetically shielded room in which mMEG activity was recorded while participants performed the experiment. To record vertical and horizontal eye movements, electrooculographic activity was obtained. Electrode impedance was kept $<10$ $\mathrm{k} \Omega$. For the data acquisition, a whole-head, 102-channel magnetometer array (Elekta Neuromag TRIUX) with internal helium recycler has been used. The MEG system contains 102 sensor fields, each equipped with one magnetometer measuring the normal field component and two orthogonally oriented planar gradiometers for measuring the gradient components. The participants sat in an upright position underneath the MEG "helmet." MEG data were sampled at $2000 \mathrm{~Hz}$ with a bandpass filter from DC to $660 \mathrm{~Hz}$.

First, we tested whether participants discount more in the OTHER condition compared with the SELF condition comparing log-transformed discount parameters between conditions. Participants were instructed to withhold decision until the fixation point turned red $(3 \mathrm{~s} \pm$ $200 \mathrm{~ms}$ following presentation of choice options; sufficiently long to exceed mean reaction time in the first experiment) to eliminate the impact of button presses (see Fig. $1 C$ ). In the eye tracking experiment, we forced participants to shift gaze and hence their attentional focus. Here, to suppress eye movements, choice options were presented on the screen spanning an area of $3 \times 12 \mathrm{~cm}$ (horizontal visual angle of $6.8^{\circ}$ and vertical visual angle of 
A

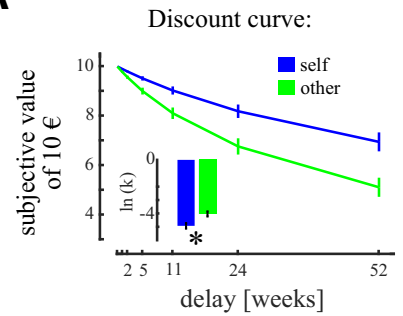

B

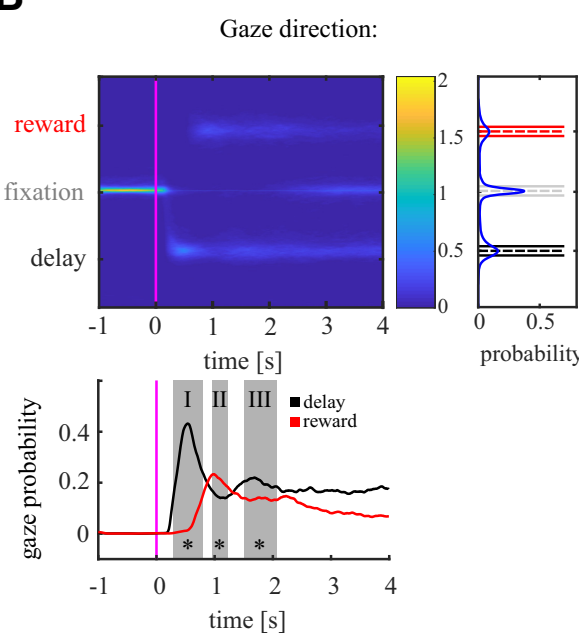

C
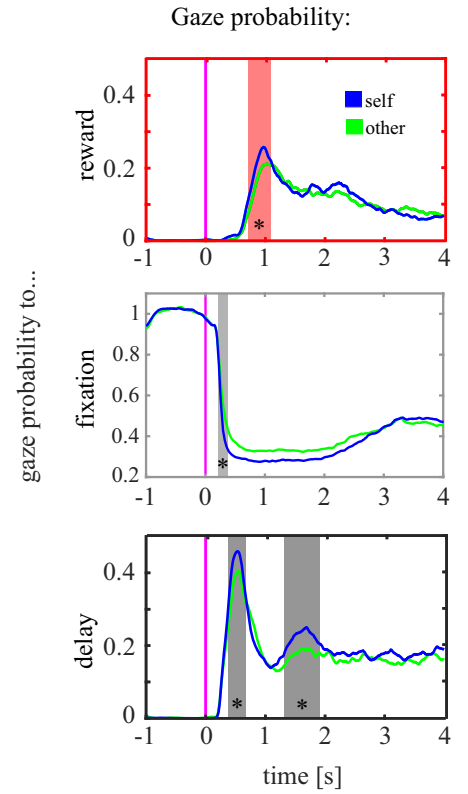

D

Gaze-Discountig correlation:

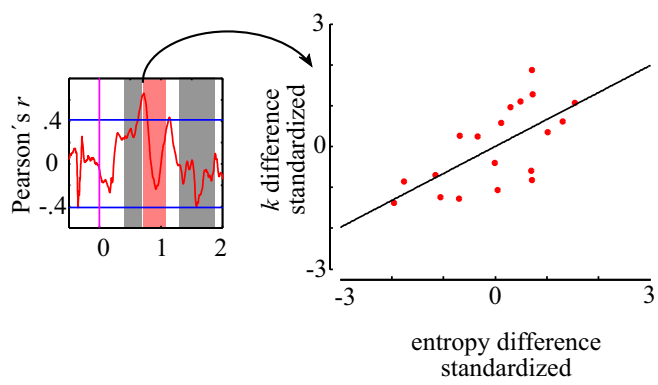

Figure 4. Eye tracking results. $A$, Stronger discounting in the OTHER condition as indicated by differences in discount parameters (small inset). Error bars indicate SEM. $\boldsymbol{B}$, Color-coded gaze probability as a function of time to choice option onset ( $x$ axis) averaged across all participants and conditions. $y$ axis indicates the location of reward, fixation, and delay presented on the screen. Yellow band before stimulus onset (magenta vertical line) indicates that participants looked at the fixation cross. Following stimulus onset, participants directed gaze to delay and reward as indicated by light green and light yellow streaks across time. Right, Average across time. Peaks (dashed lines) correspond to exact locations on the screen of reward, fixation, and delay. Bell-shaped averaged probability values indicate that participants inspected reward and delay with spatial variability. Probability values within inflection points (solid lines) were averaged, leading to time series representing gaze probability to delay, reward, and fixation. Bottom, A consistent chronology of first delay and then reward inspection. Black and red lines indicate probability to direct gaze to delay and reward, respectively, averaged across participants. First gray shaded area represents the interval in which participants gazed to delay more than to reward as indicated by a significant $t$ value; the second gray shaded area, vice versa. C, Time-varying probability to direct gaze to fixation in both conditions averaged across participants. Gray shaded area represents the temporal interval, with significant difference between SELF (blue) and OTHER (green) condition indicating that participants disengaged slower from fixation in the OTHER condition. Bottom, Gaze direction to the delay (black frame) separately for both conditions. Top, Gaze direction to reward (red frame) separately for both conditions. Gray shaded areas represent intervals of significant differences between conditions, indicating that both delay and reward were inspected more closely in the SELF compared with the OTHER condition. $\boldsymbol{D}$, Correlation of differences of gaze entropy and difference of discount parameter between conditions at the time point shown in the left small diagram. Greater differences in gaze entropy are correlated with greater differences in discounting.

$\left.1.79^{\circ}\right)$. This allows us to assess potential intervals when delay and reward are within the attentional focus, ruling out the possibility that differences in MEG activity result from eye movements. Furthermore, all trials with activity $>2$ SDs of all electro-oculographic trials were rejected.
Preprocessing. We used MATLAB 2013b (The MathWorks) for all offline data processing. All filtering (see below) was done using zero phase-shift IIR filters. First, we used an absolute threshold of $300 \mathrm{fT}$ to discard signal epochs of excessive, nonphysiological amplitude. We then visually inspected all data, excluded epochs exhibiting excessive muscle activity, as well as time intervals containing artifactual signal distortions, such as signal steps or pulses. We refrained from applying artifact reduction procedures that affect the dimensionality and/or complexity of the data (e.g., independent component analysis). The raw signal of all remaining epochs was filtered between 1 and $275 \mathrm{~Hz}$. A notch filter was applied to remove line noise $( \pm 2 \mathrm{~Hz}$ around the first 5 harmonics) before filtering in specific frequency bands (see below).

Data analysis. Discounting differences. First, we compared $\ln (k)$ parameters across participants to test whether participants discounted differently between conditions (see Fig. 5A).

Choice options related to amplitude modulation. Next, we tested whether brain activity shows significant amplitude modulation to presentation of choice options. For each trial $(-2 \mathrm{~s}$ to $6 \mathrm{~s}$ around stimulus onset sufficiently long to prevent any edge effects during filtering), we bandpass filtered each electrode's time series at 37 frequency bands (log-spaced between 1 and $330 \mathrm{~Hz}$ ) with a bandwidth of $15 \%$ of the center frequency. We obtained the analytic amplitude $A_{f}(t)$ of each frequency $f$ by Hilbert-transforming the filtered time series. We smoothed the time series such that the amplitude value at each time point $n$ is the mean of $10 \mathrm{~ms}$ around each time point $n$. We then baseline-corrected the brain activity by subtracting the mean activity from the -1 to $0 \mathrm{~s}$ preceding the stimulus onset in each trial of each magnetometer.

We then identified stimulus-responsive frequency bands showing a significant amplitude modulation in each frequency band following the onset of choice display. We first averaged $A_{f}$ across all trials, magnetometers, and participants, resulting in one amplitude time series for each frequency. We then calculated the average baseline activity $\bar{B}_{f}$ across the $500 \mathrm{~ms}$ preceding the stimulus onset. For each frequency band activity, we subtracted $\bar{B}_{f}$ from the activity modulation $\bar{A}_{f}$ averaged across the $3 \mathrm{~s}$ following the stimulus onset. To control the significance threshold for multiple comparisons, the difference between $\bar{B}$ and $\bar{A}$ was compared against an empirical distribution derived from randomly shifted time series $\left(N_{\text {permutations }}=1000\right)$. In each iteration, time series of each channel (circular shift of the entire trial time series) separately and new (surrogate) trial averages $(\bar{B}$ and $-A)$ were calculated from the shifted trials. Frequency bands exceeding the 97.5 th or below the 2.5 th percentile of the frequency specific surrogate $\bar{B}_{f} \bar{A}_{f}$ distribution (see Fig. $5 B$, dashed 
gray lines) were classified as showing a significant amplitude modulation following presentation of choice options.

The previous analysis informed us which frequency band showed a significant modulation to presentation of choice options. Next, we tested which channels contributed significantly to the stimulus response modulation in frequency bands with significant amplitude modulation. Hence, we filtered raw time series in the broader frequency bands showing significant amplitude modulation found in the previous analysis. We first averaged $A_{f}$ across all trials and participants and repeated the analysis as outlined above. Magnetometer's signals exceeding the two-sided 95th percentile of the surrogate $\bar{B}_{f} \bar{A}_{f}$ distribution were classified as showing a significant amplitude modulation following presentation of choice options (see Fig. 5B).

Amplitude modulation with delay. In the next step, we tested whether these frequency bands code objective values and hence showed amplitude modulation as a function of delay and/or reward. First, we grouped trial activity recorded at magnetometers with significant amplitude modulation according to the six different delays and averaged across all participants. This was done since the six different delays were the same in all participants. This leads to six new time series for each magnetometer, each representing the mean activity modulation to one of the six delays. At each time point, we linearized amplitude differences between these 6 time series by assigning a rank value to each of the 6 amplitude values ( 1 being the lowest amplitude and 6 representing the highest amplitude). Integer ranks can help to stabilize effects, which can be obscured because of these fluctuations. This was used to identify temporal intervals. To corroborate our hypothesis, statistical tests were conducted using the real data. Next, we tested whether these ranks as a proxy for the amplitude values correspond with the delays. A rank order matching the number of delay ( 1 [shortest delay] to 6 [longest delay]) would indicate that a given frequency band responds with a gradually increasing amplitude modulation to a gradually increasing delay. To test this, we used a linear least square fit to the rank values at each time point. This results in a new slope time series with positively/negatively highest slopes when amplitude modulation varies with delay and slopes at $\sim 0$ when amplitude is not modulated by the delay. The level of significance was corrected for multiple comparisons by comparing each slope value against a surrogate distribution. This surrogate distribution was constructed by randomly reassigning the labels (delay 1-6) to the six time series in 1000 permutations for each channel. This leads to 1000 surrogate slope values. Frequency bands exceeding the two-sided 95th percentile of the surrogate distribution were classified as showing a significant correlation of amplitude and delay. To assess differences between frequency bands, we used a time point-by-time point oneway ANOVA to test for differences of slope values across magnetometers. We determined the empirical significance threshold for $F$ values by randomly reassigning the frequency band labels in 1000 permutations of the same time point-by-time point ANOVA. Last, in the temporal interval of significant amplitude-by-delay covariation, we used a one-way ANOVA to test for differences of amplitude values between

A

C

D

$\mathbf{E}$ conditions.
Discount curve:

B
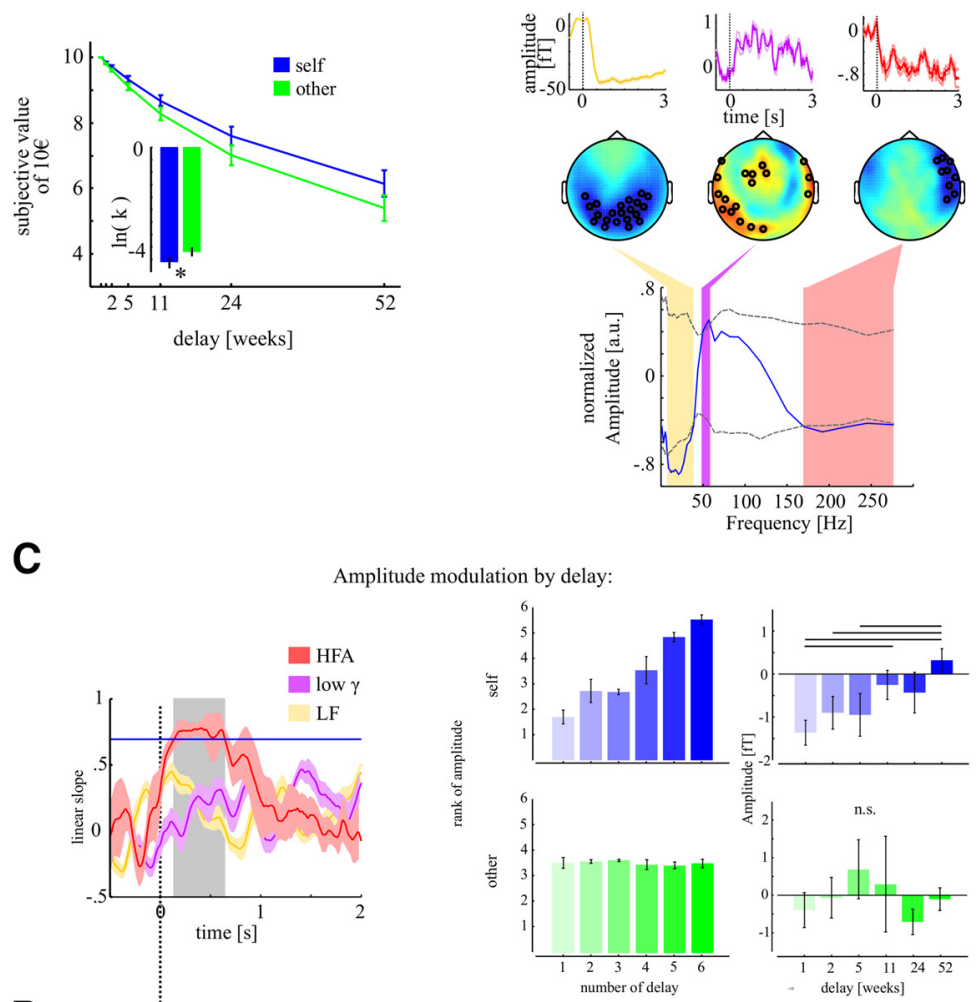

Correlation of amplitude with reward:

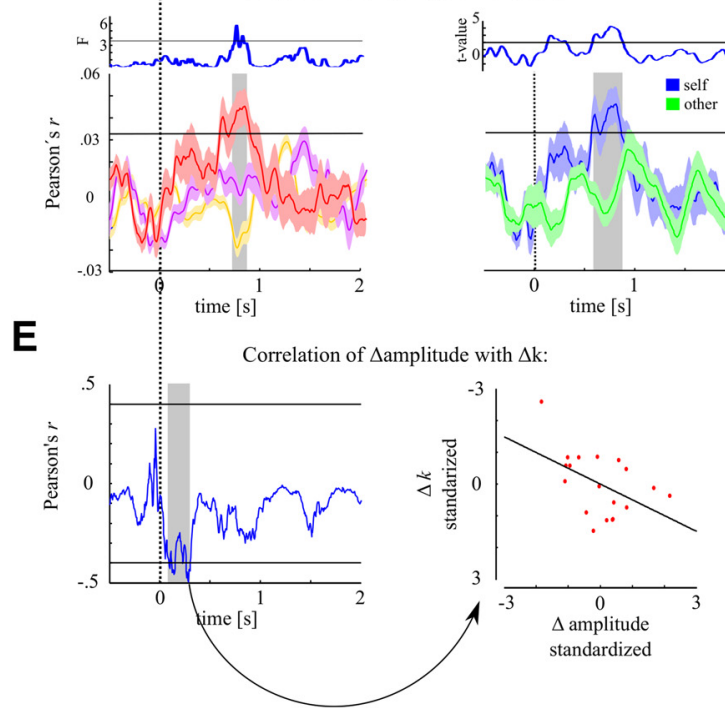

Figure 5. MEG results. $\boldsymbol{A}$, Behavioral results. $\boldsymbol{B}$, Amplitude modulation in three different frequency bands with topographical distributions. Two gray dashed lines indicate the upper and lower confidence interval (obtained from a permutation test) and hence distinguishes the frequency bands with significant amplitude decrease (below the lower gray line) from frequency band with significant amplitude increase (above the upper gray line). In each frequency band, we determined MEG sensors with significant amplitude modulation over baseline based on a permutation procedure. C, Only HFA shows correlation with delay. Differences in amplitude modulation as a function of delay was only found in the SELF but not in the OTHER condition. Error bars indicate SEM. $\boldsymbol{D}$, In a temporal interval following the coding of the delay, we found significant correlation with reward information only in the high frequency band and exclusively in the SELF condition. $E$ : Differences in amplitude modulation between conditions were correlated with differences of discounting between

6 delays across participants. The same analysis was conducted in the OTHER condition (see Fig. 5C).

Correlation with reward. To test whether amplitude modulation varied as a function of the reward, we correlated the trial-to-trial variation of reward with the trial-to-trial variation of amplitude averaged across 
Table 1. Participants characteristics and exclusion criteria

\begin{tabular}{lllccc}
\hline & $n$ (total participants) & Age (mean \pm SD) & Gender (m/f) & MEG artifacts $>30 \%$ & $n$ (final sample size) \\
\hline Reaction time & 22 & $24.10 \pm 5.16$ & $9 / 13$ & - & 6 \\
Eye tracking & 20 & $22.58 \pm 2.06$ & $10 / 10$ & -6 & 16 \\
MEG & 24 & $26.17 \pm 5.16$ & $17 / 7$ & 1 & 19 \\
\hline
\end{tabular}

MEG sensors showing significant amplitude modulation at each time point. This analysis was done in each participant since monetary reward values depended on individual decisions and consequently differed across participants. This results in a Pearson's $r$ time series for each participant. The level of significance was corrected for multiple comparisons by comparing each $r$ value against a surrogate distribution. This surrogate distribution was constructed by randomly reassigning the amplitude values of one participant to the discount values of another participant in 1000 permutations. This leads to 1000 surrogate $r$ value time series. Significance criterion was an $r$ value with $p<0.025$ within the surrogate distribution of all $r$ values (see Fig. 5D).

Correlation of MEG response and discount differences. Finally, we assessed whether differences in amplitude modulation could explain differences of discounting behavior between conditions. We averaged in frequency bands showing significant amplitude modulation across MEG sensors representing objective choice options in both conditions and calculated the difference time series $\left(\Delta_{\text {amplitude }}\right)$ for each participant. At each time point, we calculated Pearson's $r$ between $\Delta_{\text {amplitude }}$ and $\Delta_{\mathrm{k}}$ denoting the difference in discount parameter in each participant. The level of significance was corrected for multiple comparisons by comparing each $r$ value against a surrogate distribution. This surrogate distribution was constructed by randomly reassigning the amplitude values of one participant to the discount values of another participant in 1000 permutations. This leads to 1000 surrogate $r$ value time series. Significance criterion was an $r$ value with $p<0.025$ within the surrogate distribution of all $r$ values (see Fig. 5E).

\section{Results}

In all experiments, participants were asked to choose between a LL amount of $10 €$ at a variable delay $D(1,2,5,11,24$, or 52 weeks; presented in pseudorandom order) and SS (now) reward (for a detailed description of the paradigm, see Materials and Methods; Fig. 1). Participants made 10 choices for each delay in pseudorandom order, while SS was adjusted according to the previous response to reach equivalent LL and SS options (see Materials and Methods; Table 1).

\section{Decision time experiment}

In the first experiment, we tested 22 participants ( 13 female; mean age 24.1 years; $S D=5.16$; all righthanded with normal or corrected-to-normal vision) (1) for differences in decision time and (2) whether they discounted differently between both conditions (analysis steps are explained in more detail in Materials and Methods). We calculated a CI, which parameterizes how evenly participants chose both options. Six participants strongly preferred one choice option (see Materials and Methods; $C I \geq 0.66$ ), which means that one option was chosen 5 times as often as the other one and were excluded. The remaining participants did not differ on average in their $\mathrm{CI}\left(\mathrm{CI}_{\mathrm{SELF}}=0.21, \mathrm{SD}=0.15 ; \mathrm{CI}_{\mathrm{OTHER}}=0.26, \mathrm{SD}=0.14\right.$; $t_{(15)}=1.5 ; p=0.15$; Fig. $\left.2 A\right)$ and in their decision time $\left(\mathrm{RT}_{\mathrm{SELF}}=2.36\right.$ $\mathrm{s}, \mathrm{RT}_{\mathrm{OTHER}}=2.34 ; t_{(15)}=0.16 ; p=0.8$; Fig. $3 A$ ). However, they discounted stronger in the OTHER condition $\left(t_{(15)}=2.3 ; p=0.03\right)$ as indicated by higher discount parameters (mean $\ln \left(k_{\text {OTHER }}\right)=-3.7$; $\mathrm{SD}=0.23$; mean $\ln \left(k_{\text {SELF }}\right)=-4.3 ; \mathrm{SD}=0.29$; Fig. $\left.3 B\right)$.

\section{Eye tracking experiment}

To further assess OTHER condition discounting effects, we compared the discounting parameters $\ln (k)$ (the natural logarithm of the $k$ parameter adjusted throughout the experiment) in a second group $(N=20,10$ female; mean age 22.58 years; $S D=2.06)$. Eye tracking data (time series of vertical eye movements between reward presented above and delay below fixation cross) were used to characterize temporal dynamics of evaluation of delay and monetary reward. Analysis steps are explained in more detail in Materials and Methods. In general, we tested for differences in discounting behavior (see Discounting differences), whether participants showed on average a consistent chronology of delay and reward evaluation (see Intervals of option evaluation), whether participants evaluated choice options differently in both conditions (see Differences in option evaluation). These analyses test for stability choice option evaluation (gaze entropy; reflects the predictability or stability of gaze direction). In the final step, we tested whether discounting differences can be explained by differences in choice evaluation (see Correlation of gaze entropy and decision).

\section{Discounting differences}

One participant was excluded because of his strong bias toward one option $(C I \geq 0.66)$. The remaining participants showed on average no difference in $\mathrm{CI}\left(\mathrm{CI}_{\mathrm{SELF}}=0.24, \mathrm{SD}=0.17 ; \mathrm{CI}_{\mathrm{OTHER}}=\right.$ $0.15, \mathrm{SD}=0.11 ; t_{(18)}=1.7 ; p=0.11 ;$ Fig. $\left.2 B\right)$. Replicating Experiment 1, participants discounted stronger in the OTHER condition $\left(t_{(18)}=3.5 ; p=0.0025\right.$; Fig. $\left.4 A\right)$ as indicated by higher discount parameters $\left(\right.$ mean $\ln \left(k_{\text {OTHER }}\right)=-3.9$; $\mathrm{SD}=0.84$; mean $\ln \left(k_{\text {SELF }}\right)=-4.8 ; \mathrm{SD}=0.62$; Fig. $\left.4 A\right)$.

\section{Intervals of option evaluation}

We tested the chronological order of the inspection of delay and reward. Participants tended to inspect delay first (Fig. 4B, I; significant difference to reward between $182 \mathrm{~ms}$ and $816 \mathrm{~ms}$; $t_{\text {crit }}= \pm 2.05 ; t_{\max }=12.35$ at $\left.536 \mathrm{~ms} ; p<0.000001\right)$. Participants then inspected the reward (Fig. 4B, II) between 998 and $1201 \mathrm{~ms}$ indicated by higher probability for gaze at reward compared with delay $\left(t_{\max }=2.45\right.$ at $\left.1102 \mathrm{~ms} ; p=0.01\right)$. Third, they returned to the delay (Fig. $4 B$, III) between 1447 and $2110 \mathrm{~ms}\left(t_{\max }=3.68\right.$ at $1628 \mathrm{~ms} ; p=0.002$ ) with higher probability for gaze at delay compared with reward.

\section{Differences in option evaluation}

Here we tested for temporal differences of inspection as a function of our experimental conditions. We found that participants more closely inspected the delay (I) in the SELF condition (Fig. $4 C$, bottom) in the temporal interval from 367 to $626 \mathrm{~ms}\left(t_{\text {crit }}= \pm 2.075\right.$; $t_{\max }=2.8$ at $\left.416 \mathrm{~ms} ; p=0.0038\right)$. Second, we found that participants more closely inspected the reward (II) in the SELF condition (Fig. $4 C$, top) in the temporal interval from 686 to $1032 \mathrm{~ms}\left(t_{\max }=\right.$ 2.95 at $925 \mathrm{~ms} ; p=0.0026)$. Third, when participants returned to the delay information, they also more closely inspected the delay (III) in the SELF condition between 1434 and $1634 \mathrm{~ms}\left(t_{\max }=2.36\right.$ at $1686 \mathrm{~ms} ; p=0.012$ ).

\section{Correlation of gaze entropy and decision}

In the last step, we tested whether differences in gaze entropy (how stable participants looked at choice options) between conditions correlate with differences in discount parameter between 
conditions. We found a significant correlation (critical $r$ value was \pm 0.41 ) between differences in discount parameter and differences in gaze entropy between 628 and $778 \mathrm{~ms}\left(r_{\max }=0.66\right.$ at $722 \mathrm{~ms}, \beta=89 \%$; $p=0.0025$; Fig. $4 D$ ).

\section{MEG experiment}

In a third group of participants $(N=24 ; 7$ female; mean age: 26.17 years; $\mathrm{SD}=5.16$ years), we assessed discounting behavior (see Discounting differences) using MEG activity across specific frequency bands (see Choice options-related amplitude modulation). We then tested whether these specific frequency bands represent the delay (see Amplitude modulation with delay), and/or the reward (see Correlation with reward) and whether differences of MEG activity between conditions correlate with differences in discounting behavior (see Correlation of MEG response and discount differences). Analysis steps are explained in more detail in Materials and Methods.

\section{Discounting differences}

Five participants were excluded since $>30 \%$ of trials had to be rejected because of artifacts (we recorded only 60 trials in each condition). None of the remaining participants had to be rejected because of a strong bias toward one option $(C I \geq 0.66)$. The remaining participants did not differ with respect to CI $\left(t_{(18)}=\right.$ $1.34, p=0.2)$ but show differences in discounting $\left(t_{(18)}=2.85\right.$, $p=0.01)$ consistently with the other two groups (Fig. 5A).

\section{Choice options-related amplitude modulation}

We found a low-frequency band (LF: 6-35 Hz) and high-frequency band (HF: 150-275 Hz) with significant amplitude decrease compared with baseline bilaterally located over occipital cortex and over right frontotemporal cortex, respectively (Fig. $5 B$ ). Additionally, we found $\gamma$ frequency $(50-70 \mathrm{~Hz})$ increase over baseline following stimulus onset in a central and occipital ROI (Fig. 5B).

\section{Amplitude modulation with delay}

Only HF amplitude varied with the delay information (critical slope value: 0.69 ) between 138 and $643 \mathrm{~ms}$ (slope $_{\max }=0.78$ at $411 \mathrm{~ms} ; p=0.03$; Fig. $5 C$ ). In the SELF condition, we found differences in amplitude modulation depending on the delay presented in this interval $\left(F_{(5,108)}=2.4 ; p=0.039\right)$. Post hoc tests revealed a significant difference between 1 and 11 weeks $(p=0.04)$ and 1 and 52 weeks $(p=0.0003), 2$ and 52 weeks $(p=0.01)$, and 5 and 52 weeks $(p=0.03)$. The OTHER condition exhibits no significant differences $\left(F_{(5,108)}=0.5\right)$. In addition, we found a highly significant interaction between the OTHER and $\operatorname{SELF}$ condition $\left(F_{(5,216)}=9.06 ; p<0.00001\right)$.

\section{Correlation with reward}

The HF amplitude was correlated with reward between 576 and $876 \mathrm{~ms}\left(r_{\max }=0.05 ; p=0.0009\right.$ at $816 \mathrm{~ms}$; Fig. $\left.5 D\right)$, which is also corroborated by a significant difference between frequency bands between 741 and $826 \mathrm{~ms}\left(F_{\text {crit }}=3.6 ; F_{\max }=5.8\right.$ at $756 \mathrm{~ms}$; $p<0.00001)$. Importantly, in the OTHER condition, HF amplitude was not correlated with the monetary reward. Next, we compared directly the correlation values between both conditions at each time point. Figure $5 D$ shows the resulting $t$ value time series. We found that correlation values differed between conditions (561 and $861 \mathrm{~ms} ; t_{\max }=4.3$ at $741 \mathrm{~ms} ; p<0.000001$ ). This analysis revealed that the significant reward correlation in the SELF condition is not only significant but also larger than in the OTHER condition.

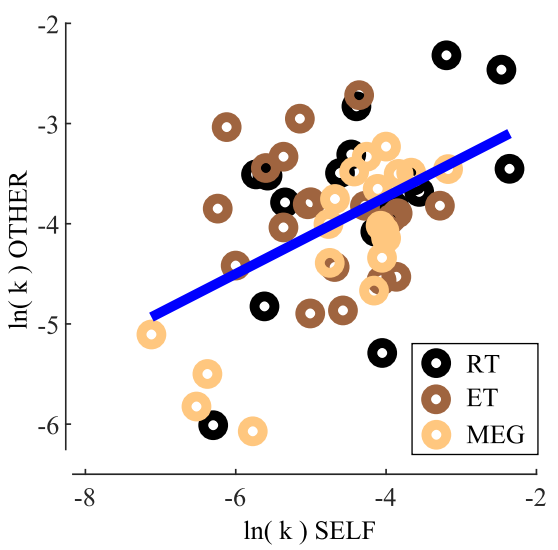

Figure 6. Follow-up analysis. Correlation of discount parameters between conditions across all three experiments: reaction time (RT), eye tracking (ET), and MEG.

\section{Correlation of $M E G$ response and decision}

We found that the differences of the HFA predicted differences in discounting behavior between 96 and $310 \mathrm{~ms}\left(r_{\min }=-0.5\right.$ at $286 \mathrm{~ms} ; p=0.01, \beta=0.64$; Fig. $5 E$ ). Although significant, the power of this analysis is relatively low compared with the correlation analysis in the eye tracking paradigm and the follow-up analysis (see below). Moreover, the individual differences analysis was conducted in a time-resolved manner. Hence, there is considerable variation over time and only for a specific temporal interval we can reliably define a correlation. We hypothesize that future studies, designed to specifically test for an intermediate correlation strength using more participants, might be able to delineate the temporal evolution of individual differences between MEG responses and decisions with higher accuracy.

\section{Follow-up analysis}

We tested whether discounting behavior in the SELF condition was correlated with the OTHER condition across the three different groups. Differences in discounting parameters accompanied by a significant correlation indicate a similar baseline mechanism for decision-making, which utilizes a different level of objective information. We found, across all experiments, In ( $k$ ) parameters correlated between both conditions $(r=0.48$; $p=0.0003, \beta>0.95$; Fig. 6).

\section{Discussion}

Studies on DD have focused on the representation of subjective value signals by contrasting differential activation associated with smaller and sooner versus larger and longer choices (McClure et al., 2004; He et al., 2012; Kim et al., 2012; Cooper et al., 2013; Peper et al., 2013; van den Bos and McClure, 2013; van den Bos et al., 2014, 2015). Impulsivity, the choice of SS, is associated with preferring sooner rewards compared with later rewards or to insufficiently considering objective alternatives (Ainslie, 1975; Myerson et al., 2003; Olson et al., 2007).

We tested whether subjective value (by contrasting self-referential decisions with prosocial decisions) counteracts impulsivity. There is considerable debate whether humans are truly prosocial. In intertemporal choices, it is unclear whether either a higher reward or an earlier gratification is prosocial. Previous studies showed that participants put in more effort when acts benefited themselves (Lockwood et al., 2017). In our experimental settings across different groups, participants showed impulsive decisions 
(discounted less in self-referential compared with prosocial decisions) but did not show condition decision time differences seen in impulsive actions (Cho et al., 2010; Wang et al., 2016; Yates et al., 2016). Hence, we hypothesized that differences in discounting result from differences in depth of evaluation of choice options. Therefore, prosocial decisions provide the opportunity to test how choice option integration in human participants is represented and whether information, which pertains to ourselves, is processed differently compared with prosocial decisions.

In order to elucidate the temporal evolution of the option integration process itself, we compared patterns of attentional reallocation (eye tracking) and choice option integration (MEG). In our eye tracking experiment, the increased spatial distance between choice options on the screen enhanced the effort to move the eyes, corroborating predictions of less effort in the prosocial condition (social apathy) (Lockwood et al., 2017). Patterns of choice option evaluation were similar explaining why decision times are not different but gaze stability differed between conditions, explaining interindividual differences in discounting behavior. We hypothesize that less attentional orientation limits representing and integrating choice options. MEG was recorded in a separate experiment with minimal spatial extent of choice option presentation on the screen, minimizing eye movement contamination of the temporal evolution of choice option integration allowing replication of behavioral results in two independent groups. To compare eye tracking with MEG results, we constructed time series representing the probability to look at a given spatial point as a function of time. We observed that intervals of attentional shift to the delay and reward in the eye tracking experiment were paralleled by intervals of representation of delay and reward in MEG in the HFA.

Intertemporal choices in DD experiments refer to the tradeoff between benefits and costs, which can be either an increasing delay ("wait") or increasing effort ("work") to obtain the reward (Phung et al., 2019). Both can be dissociated by underlying neuronal circuits driving behavior toward reward maximization and effort minimization (Prévost et al., 2010; Massar et al., 2015; Klein-Flügge et al., 2016) accompanied by differences in discount curves with an inverted sigmoid function and hyperbolic function in effort and DD, respectively. We did not see an initial concave shape in the prosocial condition typical for effort discounting arguing against the notion that both conditions recruit different neuronal networks. Instead, we propose that differences in discounting result from differences in choice option evaluation. This provides evidence that evaluating gaze stability is a proxy of attentional direction to choice options, and predicts DD.

The MEG study showed that only high-frequency activity (HFA; 175-250 Hz) was modulated by choice options exclusively in the self-referential condition, providing evidence that activity distributed in MEG sensors over frontotemporal regions reflects integration of delay and the reward in humans. HFA is assumed to reflect nonrhythmic synaptic activity (Buzsáki et al., 2012) and is a key marker of cortical activation (Edwards et al., 2005; Ray et al., 2008). Intracranial recordings of HFA response dynamics in humans have enhanced our understanding of cortical information integration in attention, language, memory, emotion, decision-making, and motor control (Johnson et al., 2020). These studies imply that HFA acts as an index of local cortical computation (Buzsáki et al., 2012; Rich and Wallis, 2017). HFA bridges a long-standing gap to fMRI studies on DD. Power modulation in higher frequencies has been shown to explain BOLD responses better than activity in lower frequencies, which are instead thought to reflect activity in broadly distributed networks (Nir et al., 2007; Mukamel et al., 2014). The temporal precision of MEG adds to the spatial resolution of fMRI and is able to delineate mechanisms of choice option integration in time, which is in line with previous studies on humans and nonhuman primates showing that HFA captured reward-related information (Hunt et al., 2015). HFA has been regarded as a good measure of neuronal spiking (Liu and Newsome, 2006; Berens et al., 2008), consistent with the idea that HFA reflects aggregate local neuronal output (Buzsáki et al., 2012) because of high correlations between HFA and multiunit activity. Both can distinctively be localized in granular/infragranular and supragranular layers, respectively, in $\mathrm{V} 1$ and $\mathrm{A} 1$ in monkeys and PFC in humans. Supragranular HFA contributes significantly more to the surface field potential than deeper layers, and it is argued that HFA may contain a substantial representation of input from cortical feedback pathways (Leszczyński et al., 2020). Recent single-neuron studies in monkeys provide insight into the neural mechanism for the estimation of interval time (Brody et al., 2003). Singleunit activity is modulated by the amount of an expected reward (Leon and Shadlen, 1999; Wallis and Miller, 2003) and encodes the relative reward value (Tremblay and Schultz, 1999; Cai et al., 2011). Furthermore, single-neuron recordings in pigeons showed that neural delay activity was modulated by increasing delay length and additionally covaried with expected reward amount (Kalenscher et al., 2005).

The right dlPFC, associated with executive and control functions, potentially represents choice options (Bickel et al., 2009; Achterberg et al., 2016) and response selection among the most advantageous (Ho et al., 2016). Our results indicate that self-referential decisions are characterized by a response selection suppression mitigating impulsive decisions. Activity in the medial and right dlPFC is also positively correlated with self-risk ( $\mathrm{Hu}$ et al., 2017) and cathodal transcranial direct current stimulation (tDCS), associated with inhibition, reduces impulsivity and risky behavior in Parkinson patients (Benussi et al., 2017). Thus, lower levels of activity are associated with less impulsivity, which is in line with a relative HFA reduction during SELF compared with OTHER decisions in our study. Importantly, we did not evaluate the activity level of impulsive versus patient decisions but the neural activity accompanying choice option presentation preceding these decisions. The disruption of right dlPFC with low-frequency repetitive transcranial magnetic stimulation reduces emotional weight during decision-making in social contexts (Tassy et al., 2012), indicating that the dlPFC actually integrates objective choice options. These findings are controversial since the impact of tDCS on the right dlPFC is complex (low risk aversion in gain frames after tDCS but high-risk aversion in loss frames after stimulation) (Ye et al., 2016). Other studies showed that the right dlPFC mediates action value comparisons in value-based decision-making (Morris et al., 2014) and plays a causal role in the computation of values of choices (Camus et al., 2009).

Mapping of sources to sensors is ill-posed in noninvasive recordings. Hence, a multitude of different source combinations could generate the field pattern. Only direct intracranial recordings can reliably distinguish anatomic localizations. Furthermore, we operationalized prosocial acts by taking perspective of the best friend. It could be argued that participants did not perform genuine prosocial acts since they did not directly benefit others because of the hypothetical outcomes. How real rewards, directly paid to others, influence prosocial 
decisions in intertemporal choices should remain to be determined. Moreover, future studies can test for an intermediate correlation strength using more participants to delineate with higher accuracy the temporal evolution of correlation between MEG responses and decisions.

Intertemporal behavior has emphasized trait-like variance (Luo et al., 2014). We found correlation between the discount parameter in the SELF and OTHER condition arguing in favor of an individual disposition to discount delayed values. In sum, we argue that impulsivity does not result from oversensitivity to one option but a lack of attentional allocation to choice options. The HFA measured over the right frontotemporal cortex shows broadband amplitude modulation only when decisions have a high value for the self but not during anonymous prosocial decisions. Intervals of delay and reward representation match intervals of gaze toward delay and reward. In sum, our results highlight a unique role of high frequency band activity recorded over the right frontotemporal cortex representing objective values important to suppressing impulsivity.

\section{References}

Achterberg M, Peper JS, van Duijvenvoorde AC, Mandl RC, Crone EA (2016) Frontostriatal white matter integrity predicts development of delay of gratification: a longitudinal study. J Neurosci 36:1954-1961.

Ainslie G (1975) Specious reward: a behavioral theory of impulsiveness and impulse control. Psychol Bull 82:463-496.

Baddeley A (1992) Working memory: the interface between memory and cognition. J Cogn Neurosci 4:281-288.

Benussi A, Alberici A, Cantoni V, Manenti R, Brambilla M, Dell'Era V, Gazzina S, Manes M, Cristillo V, Padovani A, Cotelli M, Borroni B (2017) Modulating risky decision-making in Parkinson's disease by transcranial direct current stimulation. Eur J Neurol 24:751-754.

Berens P, Keliris GA, Ecker AS, Logothetis NK, Tolias AS (2008) Feature selectivity of the gamma-band of the local field potential in primate primary visual cortex. Front Neurosci 2:199-207.

Bickel WK, Pitcock JA, Yi R, Angtuaco EJ (2009) Congruence of BOLD response across intertemporal choice conditions: fictive and real money gains and losses. J Neurosci 29:8839-8846.

Brody CD, Hernández A, Zainos A, Romo R (2003) Timing and neural encoding of somatosensory parametric working memory in macaque prefrontal cortex. Cereb Cortex 13:1196-1207.

Buzsáki G, Anastassiou CA, Koch C (2012) The origin of extracellular fields and currents: EEG, ECoG, LFP and spikes. Nat Rev Neurosci 13:407-420.

Cai X, Kim S, Lee D (2011) Heterogeneous coding of temporally discounted values in the dorsal and ventral striatum during intertemporal choice. Neuron 69:170-182.

Camus M, Halelamien N, Plassmann H, Shimojo S, O’Doherty J, Camerer C, Rangel A (2009) Repetitive transcranial magnetic stimulation over the right dorsolateral prefrontal cortex decreases valuations during food choices. Eur J Neurosci 30:1980-1988.

Cho SS, Ko JH, Pellecchia G, Van Eimeren T, Cilia R, Strafella AP (2010) Continuous theta burst stimulation of right dorsolateral prefrontal cortex induces changes in impulsivity level. Brain Stimul 3:170-176.

Cooper N, Kable JW, Kim BK, Zauberman G (2013) Brain activity in valuation regions while thinking about the future predicts individual discount rates. J Neurosci 33:13150-13156.

Edwards E, Soltani M, Deouell LY, Berger MS, Knight RT (2005) High gamma activity in response to deviant auditory stimuli recorded directly from human cortex. J Neurophysiol 94:4269-4280.

Erdfelder E, Faul F, Buchner A, Lang AG (2009) Statistical power analyses using $\mathrm{G} *$ Power 3.1: tests for correlation and regression analyses. Behav Res Methods 41:1149-1160.

Fuster JM (1990) Behavioral electrophysiology of the prefrontal cortex of the primate. Prog Brain Res 85:313-314.

Goldman-Rakic PS (1992) Working memory and the mind. Sci Am 267:110117.

Green L, Myerson J (2004) A discounting framework for choice with delayed and probabilistic rewards. Psychol Bull 130:769-792.
Hare TA, Camerer CF, Rangel A (2009) Self-control in decision-making involves modulation of the vmPFC valuation system. Science 324:646648.

He JM, Huang XT, Yuan H, Chen YG (2012) Neural activity in relation to temporal distance: differences in past and future temporal discounting. Conscious Cogn 21:1662-1672.

Ho BC, Koeppel JA, Barry AB (2016) Cerebral white matter correlates of delay discounting in adolescents. Behav Brain Res 305:108-114.

Hu J, Li Y, Yin Y, Blue PR, Yu H, Zhou X (2017) How do self-interest and other-need interact in the brain to determine altruistic behavior? Neuroimage 157:598-611.

Hunt LT, Behrens TE, Hosokawa T, Wallis JD, Kennerley SW (2015) Capturing the temporal evolution of choice across prefrontal cortex. Elife 4:e11945.

Johnson EL, Kam JW, Tzovara A, Knight RT (2020) Insights into human cognition from intracranial EEG: a review of audition, memory, internal cognition, and causality. J Neural Eng 17:051001.

Kalenscher T, Windmann S, Diekamp B, Rose J, Güntürkün O, Colombo M (2005) Single units in the pigeon brain integrate reward amount and time-to-reward in an impulsive choice task. Curr Biol 15:594-602.

Kim B, Sung YS, McClure SM (2012) The neural basis of cultural differences in delay discounting. Philos Trans R Soc Lond B Biol Sci 367:650-656.

Klein-Flügge MC, Kennerley SW, Friston K, Bestmann S (2016) Neural signatures of value comparison in human cingulate cortex during decisions requiring an effort-reward trade-off. J Neurosci 36:10002-10015.

Leon MI, Shadlen MN (1999) Effect of expected reward magnitude on the response of neurons in the dorsolateral prefrontal cortex of the macaque. Neuron 24:415-425.

Leszczyński M, Barczak A, Kajikawa Y, Ulbert I, Falchier AY (2020) Dissociation of broadband high-frequency activity and neuronal firing in the neocortex. Sci Adv 6:eabb0977.

Liu J, Newsome WT (2006) Local field potential in cortical area MT: stimulus tuning and behavioral correlations. J Neurosci 26:7779-7790.

Lockwood PL, Hamonet M, Zhang SH, Ratnavel A, Salmony FU, Husain M, Apps MA (2017) Prosocial apathy for helping others when effort is required. Nat Hum Behav 1:0131.

Luo S, Ainslie G, Monterosso J (2014) The behavioral and neural effect of emotional primes on intertemporal decisions. Soc Cogn Affect Neurosci 9:283-291.

Massar SA, Libedinsky C, Weiyan C, Huettel SA, Chee MW (2015) Separate and overlapping brain areas encode subjective value during delay and effort discounting. Neuroimage 120:104-113.

Mazur JE (1987) An adjusting procedure for studying delayed reinforcement. Hillsdale, NJ: Erlbaum.

McClure SM, Laibson DI, Loewenstein G, Cohen JD (2004) Separate neural systems value immediate and delayed monetary rewards. Science 306:503-507.

McClure SM, Ericson KM, Laibson DI, Loewenstein G, Cohen JD (2007) Time discounting for primary rewards. J Neurosci 27:5796-5804.

Morris RW, Dezfouli A, Griffiths KR, Balleine BW (2014) Action value comparisons in the dorsolateral prefrontal cortex control choice between goal-directed actions. Nat Commun 5:1-10.

Mukamel R, Fried I, Malach R (2014) Coupling between neuronal firing, field potentials, and fMRI in human auditory cortex. Science 309:951954.

Myerson J, Green L, Hanson J, Scott Holt DD, Estle SJ (2003) Discounting delayed and probabilistic rewards: processes and traits. J Econ Psychol 24:619-635.

Nir Y, Fisch L, Mukamel R, Gelbard-Sagiv H, Arieli A, Fried I, Malach R (2007) Coupling between neuronal firing rate, gamma LFP, and BOLD fMRI is related to interneuronal correlations. Curr Biol 17:1275-1285.

Olson EA, Hooper CJ, Collins P, Luciana M (2007) Adolescents' performance on delay and probability discounting tasks: contributions of age, intelligence, executive functioning, and self-reported externalizing behavior. Pers Individ Dif 43:1886-1897.

Peper JS, Mandl RC, Braams BR, de Water E, Heijboer AC, Koolschijn PC, Crone EA (2013) Delay discounting and frontostriatal fiber tracts: a combined DTI and MTR study on impulsive choices in healthy young adults. Cereb Cortex 23:1695-1702.

Peters J, Büchel C (2010) Episodic future thinking reduces reward delay discounting through an enhancement of prefrontal-mediotemporal interactions. Neuron 66:138-148. 
Phung QH, Snider SE, Tegge AN, Bickel WK (2019) Willing to work but not to wait: individuals with greater alcohol use disorder show increased delay discounting across commodities and less effort discounting for alcohol. Alcohol Clin Exp Res 43:927-936.

Prévost C, Pessiglione M, Météreau E, Cléry-Melin ML, Dreher JC (2010) Separate valuation subsystems for delay and effort decision costs. J Neurosci 30:14080-14090.

Ray S, Niebur E, Hsiao SS, Sinai A, Crone NE (2008) High-frequency gamma activity $(80-150 \mathrm{~Hz})$ is increased in human cortex during selective attention. Clin Neurophysiol 119:116-133.

Rich EL, Wallis JD (2017) Spatiotemporal dynamics of information encoding revealed in orbitofrontal high-gamma. Nat Commun 8:1139.

Tassy S, Oullier O, Duclos Y, Coulon O, Mancini J, Deruelle C, Attarian S, Felician O, Wicker B (2012) Disrupting the right prefrontal cortex alters moral judgement. Soc Cogn Affect Neurosci 7:282-288.

Tremblay L, Schultz W (1999) Relative reward preference in primate orbitofrontal cortex. Nature 398:704-708.

van den Bos W, McClure SM (2013) Towards a general model of temporal discounting. J Exp Anal Behav 99:58-73. van den Bos W, Rodriguez CA, Schweitzer JB, McClure SM (2014) Connectivity strength of dissociable striatal tracts predict individual differences in temporal discounting. J Neurosci 34:10298-10310.

van den Bos W, Rodriguez CA, Schweitzer JB, McClure SM (2015) Adolescent impatience decreases with increased frontostriatal connectivity. Proc Natl Acad Sci USA 112:E3765-E3774

Wallis JD, Miller EK (2003) Neuronal activity in primate dorsolateral and orbital prefrontal cortex during performance of a reward preference task. Eur J Neurosci 18:2069-2081.

Wang Q, Chen C, Cai Y, Li S, Zhao X, Zheng L, Zhang H, Liu J, Chen C, Xue G (2016) Dissociated neural substrates underlying impulsive choice and impulsive action. Neuroimage 134:540-549.

Yates JR, Darna M, Beckmann JS, Dwoskin LP, Bardo MT (2016) Individual differences in impulsive action and dopamine transporter function in rat orbitofrontal cortex. Neuroscience 313:122-129.

Ye H, Huang D, Wang S, Zheng H, Luo J, Chen S (2016) Activation of the prefrontal cortex by unilateral transcranial direct current stimulation leads to an asymmetrical effect on risk preference in frames of gain and loss. Brain Res 1648:325-332. 